International Journal of Advanced Information Technology (IJAIT) Vol. 8, No.1/2, April 2018

\title{
Model Monitoring Physical Exercise Heart RATE USING INTERNET OF THINGS (MMPEH-IOT)
}

\author{
Ejiofor C. I and Mgbeafuluike .I .J \\ Department of Computer Science, Chukwuemeka OdumegwuOjukwu University
}

\begin{abstract}
Model Monitoring Physical Exercise Heart Rate Using Internet of Things (MMPEH-IOT) has been developed and designed with its integral functionalities exposing through the six model components: SmartHeart, WNEt, SoftAPP, Knowledgebase, Server and User interface. The model was designed using Unified Modeling Language (UML) sequence diagram, identifying objects exchanging messages. The model forecasts future benefits into smart devices and Internet of Things (IoT).
\end{abstract}

\section{KEYWORDS}

Internet of Things (IoT), Physical Exercise, Smart Devices

\section{INTRODUCTION}

The combination of activities which excite the body organ with the aim of maintaining, enhancing, supporting physical fitness and overall body wellness is seen as physical exercise (Kylasov and Gavrov, 2011). Physical usually have either a personally, physical or mental connotation which is performed at best for different reasons ranging from growth, development, preventing aging, muscular stamina, weight loss, mental balance, cardiovascular body maintenances even sexual enhancement (Stampfer et al., 2000). Physical exercises have found it usefulness in reducing momentary stress, a principal contributor for varied mental illness inclusive of depression, dementia and even Alzheimer (Pimlott, 2010). Physical exercises have also been used efficiently and vehemently in controlling and monitoring blood sugar levels, which have resulted in the prompt prevention of numerous classes of illness inclusive of diabetes and even high blood pressure (Hu et al., 2001). Succeeding health benefits, physical exercise can encourage social cultural integration, culturally education and meaningful socialization (Boecker et al., 2008).

Physical exercises are can be perceived from three main types: Aerobic exercise, which is seen as any large muscle group, causing tremendously body oxygen consumption. Aerobic exercise increases cardiovascular endurance, which may include, running jogging, brisk walking, skipping, hiking (Wilmore and Knuttgen, 2003). It may also include anaerobic exercise traced to strength and resistance training, strength and tone muscle as well as improving body strength, balance and coordination. Anaerobic exercise may include weight training, eccentric training and interval training (De Vos et al., 2005). Physical exercise could also be seen as flexibility exercise, which may include stretch; joint flexibility and muscle limber 
International Journal of Advanced Information Technology (IJAIT) Vol. 8, No.1/2, April 2018

(O'Connor et al., 2005). Physical exercise may include training that focuses on accuracy, agility, power, and speed (De Vos et al., 2005, O'Connor et al., 2005).

Although, physical exercise forecast and provides needed health benefits, it is indeed, not without associated risk usually for aerobic and anaerobic exercise. It associated risk may include cardiac arrest, muscle injuries: feet, ankles and knees. It can also incite illness like nausea, heartburn, diarrhea, and gastrointestinal bleeding. These illnesses could be traced to serious heart rate increase tremendously during physical exercises. Therefore, it is the intent of this research paper to Monitoring Physical Exercise Heart Rate Using Internet of Things (IOT).

\section{RELATED Literature}

This section is segmented with the view of identifying and reviewing previous researches on IOT, identifying their strength, weakness and identifying area of future study. This limitation provides an avenue to prosing a novel architecture or model in addressing the identified laps or gaps in researches. Tables 2.1 provide a brief review of related literatures pertaining to Internet of Things (IoT)

Table 2.1: Review of Related works on Internet of Things (IoT)

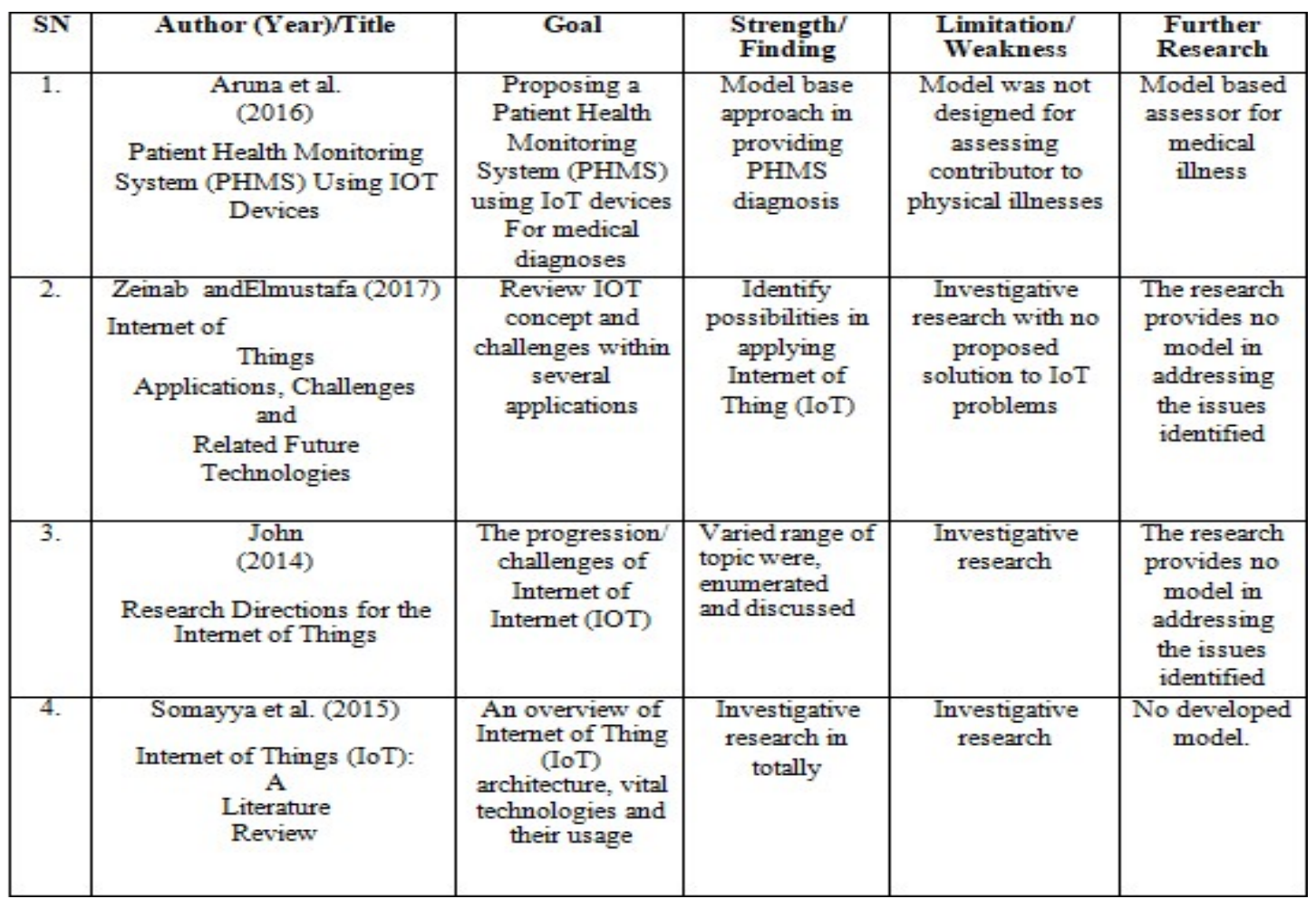

Table 2.1 identifies existing researches on Internet of Things (IoT) with notable limitations. Most of the researches focus plainly on investigative and descriptive works with little attention paid to model base solution in addressing IoTissues. Therefore base on the limitation of these literatures, 
International Journal of Advanced Information Technology (IJAIT) Vol. 8, No.1/2, April 2018

it is the intent of this research paper to monitoring physical exercise heart rate using internet of things (IoT)

\section{Monitoring Physical Exercise Heart Rate Using Internet Of ThINGS (IOT)}

The Monitoring Physical Exercise Heart Rate Using Internet of Things (MPEH-IOT) was designed with the aim of extending previous model aimed at achieving Internet of Things (IoT). Figure 3.1 graphically portray the Internet of Things Model Monitoring Physical Exercise Heart Rate.

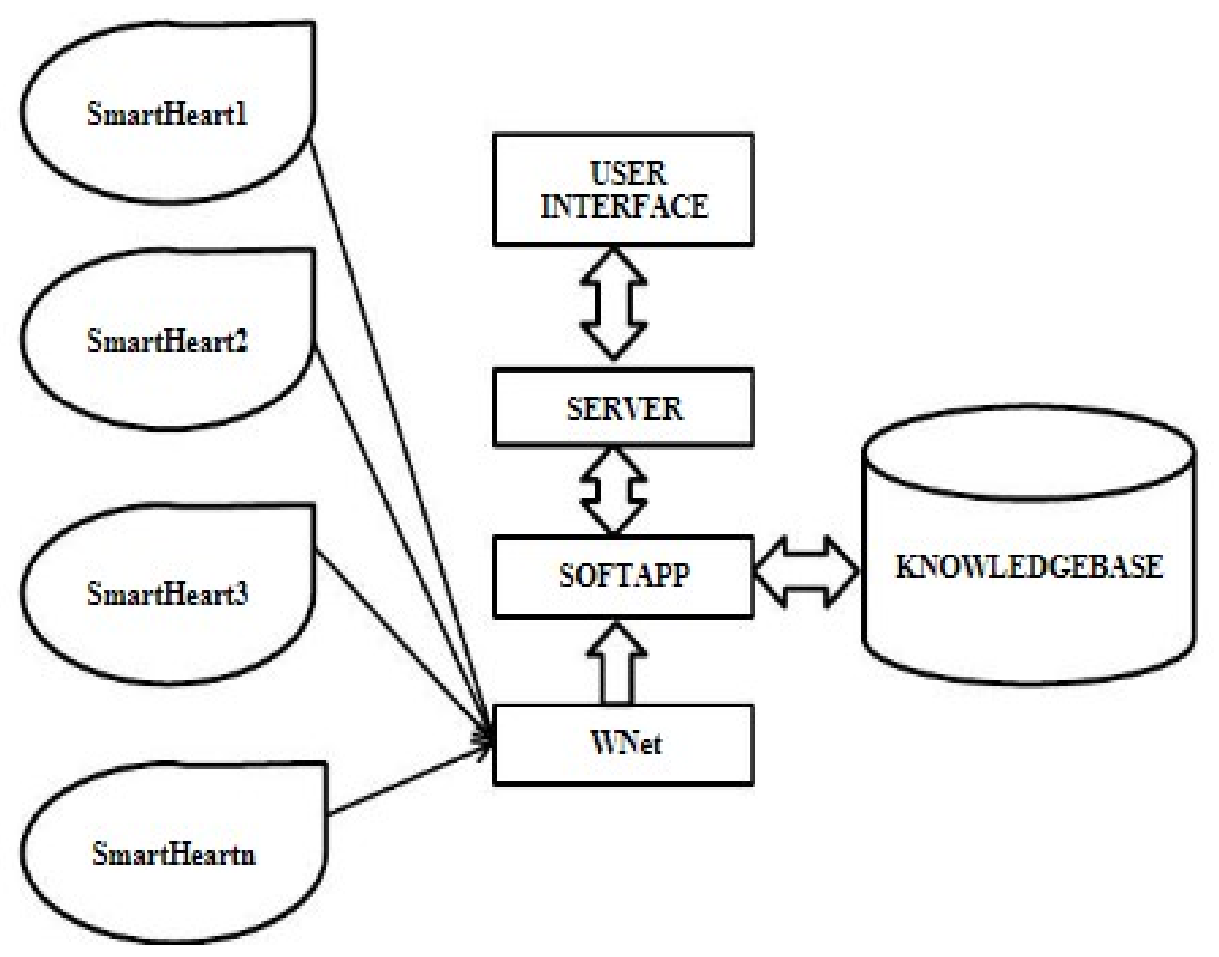

Figure 3.1: Model Monitoring Physical Exercise Heart Rate Using Internet of Things (MMPEH-IOT)

The model: Monitoring Physical Exercise Heart Rate Using Internet of Things (MMPEH-IOT) comprises of six main phases: SmartHeart, WNEt, SoftAPP, knowledgebase, server and user interface.

a. SmartHeart: The SmartHeart is known as the data collection module. This module is responsible for collecting human read heart information identified per minute and transmits it to the appropriate modules succeeding it. Therefore, all individuals engaging in such physical exercise wear the devices in returning appropriate heart rating.

b. WNet: The Wireless Network (WNet) provides the needed platform in transmitting relevant collection data from the SmartHeart to the point of analysis or evaluation. The wireless network applied for the model provide needed platform in integrating the data 
International Journal of Advanced Information Technology (IJAIT) Vol. 8, No.1/2, April 2018

collection module with the analysis and evaluation modules. Without this module, the integration and remote communication possibilities will be total impossible.

C. SoftAPP: The SoftApp provides the software application for analyzing and evaluating the collected data. The data are analyzed specifically for heart rate in determining simultaneously, if a patient is exerting his heart to much or not. This periodic analyze is stored within the knowledgebase which interface periodic with the smartheart.

d. Knowledgebase: The knowledgebase is the central repository which holds both the collected and evaluated data. This data are analyzed and stored periodically by the knowledgebase. The knowledgebase also hold the analysis rules upon which the systems runs. The two communications between the knowledgebase and the softApp, identify information integration and storage from the server and knowledgebase respectively.

e. Server: The interface serves as the intermediary between the specialized knowledgebase and the user request. This request passed through the SoftApp which is the mediator between the server and the knowledgebase. The two communications between the server and the user interface identified obtained user request and returned user request.

f. User Interface: The user interface provides an avenue for individual system user to verify and visualized evaluated and analyzed results. This result shows are presented periodically upon the rate of exercises. The two communications between the server and the user interface shows clearly.

\section{Unified Modeling Language Design (UML) FOR Model MonitoringPhysical EXercise Heart RATE USING INTERNET OF THINGS (MMPEH-IOT)}

Unified Modeling Language (UML) was employed in designing the Model: the Monitoring Physical Exercise Heart Rate Using Internet of Things (MPEH-IOT). UML been a standard modeling language provides a number of graphical tools that can be used to visualize a model from different viewpoints (Philippe, 2000 and Chris, 2000).This research paper focuses mainly on the behavioral view showing the chain of exchange. Figure 4.1 provide the sequence diagram view depicting the Monitoring Physical Exercise Heart Rate Using Internet of Things (MPEHIOT). 
International Journal of Advanced Information Technology (IJAIT) Vol. 8, No.1/2, April 2018

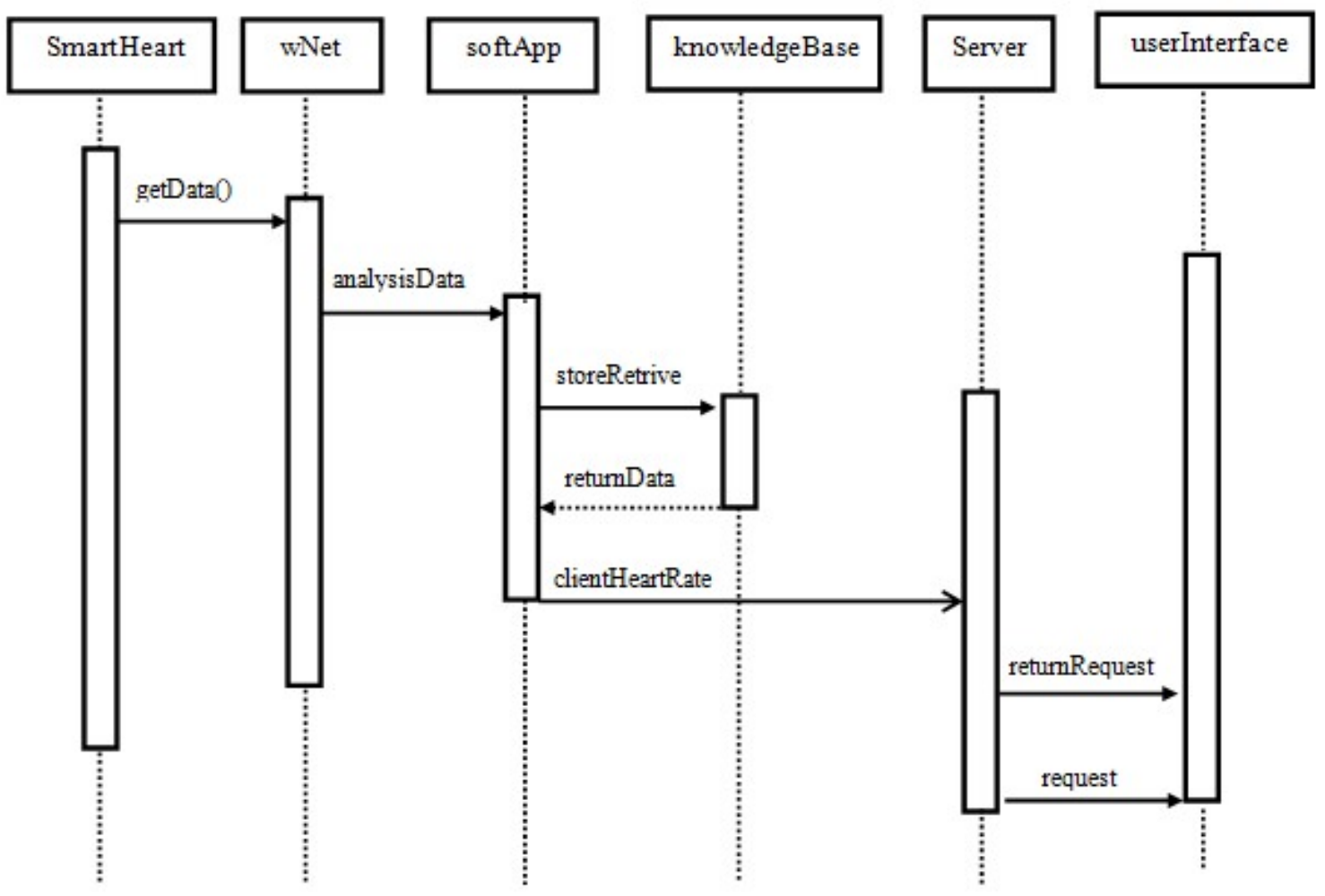

Figure 4.1:Model Monitoring Physical Exercise Heart Rate Using Internet of Things (MMPEH-IOT)

The sequence diagram portrayed on Figure 4.1 utilizing six main objects: SmartHeart, WNEt, SoftAPP, Knowledgebase, Server and User interface, in achieving heart rate monitoring. The Smartheart as integral object collect patient heart rate data which is wireless transmitted to the Wnet as an object. This information is used by the SoftApp as an object, which evaluate and analysis the data with the help of the Knowledgebase. The Server receives and transmit user request through theuserInterface object

\section{Discussion}

The Model Monitoring Physical Exercise Heart Rate Using Internet of Things (MMPEH-IOT) was designed with the aim to monitoring and evaluates the heart rate of each client engaging in meaningful of vigorous exercise. The following issues are addressed with this model:
a. Prevent Associated Health risk with vigorous exercise.
b. Determine healthy patient
c. Prevent untimely death through cardiac arrest
d. Promote healthy life

These benefits those not holistic inspire the implementation of this research paper. 
International Journal of Advanced Information Technology (IJAIT) Vol. 8, No.1/2, April 2018

\section{Conclusions}

A novel model monitoring heart rate during physical exercise was prosed using Internet of Things (IoT). The model integral six main components: SmartHeart, WNEt, SoftAPP, Knowledgebase, Server and User interface. These components holistically formed the functionalities of the model addressing the fundamental issues of health care monitoring using smart devices and Internet of Things (IoT)

\section{REFERENCES}

[1] Aruna D.S; Godfrey W.S.; Sasikumar S. (2016), Patient Health Monitoring System (PHMS) Using Iot Devices, International Journal of Computer Science \& Engineering Technology (IJCSET), Pp. 68 73.

[2] Boecker H, Sprenger T, Spilker ME, Henriksen G, Koppenhoefer M, Wagner K. J, Valet M, Berthele A., Tolle T. R (2008), The runner's high: opioidergic mechanisms in the human brain". Cereb, Cortex. 18 (11): 2523-2531.

[3] De Vos N.; Singh N.; Ross D.; Stavrinos T. (2005), Optimal Load for Increasing Muscle Power During Explosive Resistance Training in Older Adults". The Journals of Gerontology, 60A (5): 638647. doi:10.1093/gerona/60.5.638.

[4] John A. S. (2014), Research Directions for the Internet of Things, retrieved online from https://www.cs.virginia.edu/ stankovic/psfiles/IOT.pdf

[5] Hu F.B., Manson J.E., Stampfer M.J., Colditz G., Liu S., Solomon C.G., Willett W. C.; Manson; S.; Colditz; Liu S. W. (2001), "Diet, lifestyle, and the risk of type 2 diabetes mellitus in women", The New England Journal of Medicine. 345 (11): 790-797

[6] Kylasov A, Gavrov S (2011). Diversity Of Sport: non-destructive evaluation. Paris: UNESCO: Encyclopedia of Life Support Systems. pp. 462-491.

[7] O'Connor D.; Crowe M.; Spinks W. (2005), Effects of static stretching on leg capacity during cycling, Turin. 46 (1): 52-56.

[8] Pimlott N (2010). "The miracle drug". Can Fam Physician. 56 (5): 407, 409.

[9] Stampfer M.J., Hu F.B., Manson J. E., Rimm E. B., Willett W. C. ; Manson H. U; Rimm W. (2000). "Primary Prevention of Coronary Heart Disease in Women through Diet and Lifestyle". New England Journal of Medicine. 343 (1): 16-22.

[10] Wilmore J.; Knuttgen H. (2003), Aerobic Exercise and Endurance Improving Fitness for Health Benefits,ThePhysicianandSports-medicine.31(5):45.

[11] Zeinab K. A. M. and Elmustafa S. A. A. (2017), Internet of Things Applications, Challenges and Related Future Technologies, World Science News, WSN 67(2) (2017) 126 - 148 